Revue

Revue de l'histoire des religions

de Ihistoire des religions

$2 \mid 2017$

Les juifs et la nation au Moyen Âge

\title{
Les juifs visigothiques, un peuple hérétique
}

Visigothic Jews, a people of heretics

\section{Céline Martin}

\section{OpenEdition}

Journals

Édition électronique

URL : http://journals.openedition.org/rhr/8744

DOI : 10.4000/rhr.8744

ISSN : 2105-2573

Éditeur

Armand Colin

Édition imprimée

Date de publication : 1 juin 2017

Pagination : 315-335

ISBN : 978-2-200-93126-1

ISSN : 0035-1423

\section{Référence électronique}

Céline Martin, «Les juifs visigothiques, un peuple hérétique », Revue de l'histoire des religions [En ligne] 2 | 2017, mis en ligne le 01 juin 2019, consulté le 08 janvier 2021. URL : http:// journals.openedition.org/rhr/8744 ; DOI : https://doi.org/10.4000/rhr.8744 


\section{Les juifs visigothiques, un peuple hérétique}

La terminologie utilisée par les auteurs chrétiens visigothiques montre qu'ils conçoivent les juifs comme un peuple descendant d'un même ancêtre et uni par les liens du sang. Les qualifications religieuses qui leur sont appliquées se réfèrent à leur choix, erroné mais rectifiable, et ne sont donc pas au fondement de la définition de leur groupe. Ce «choix» est le principal argument qui permet de les qualifier d'hérétiques, au sens le plus large du mot, qui en comporte plusieurs; en revanche, on ne relève dans les sources aucun rapprochement entre juifs et païens. Le dernier livre du code de loi visigothique réprime d'un même élan hérétiques et juifs, peut-être parce qu'ils sont tous considérés, dans la seconde moitié du vII siècle, comme des chrétiens défectueux.

\section{Visigothic Jews, a people of heretics}

The terminology used by Visigothic Christian authors shows that they saw the Jews as a people united by blood and descended from one ancestor. Religious characterisations applied to them refer to their choice, a wrong but rectifiable one, which therefore doesn't stand at the root of their definition as a group. This "choice" is the main argument for calling them heretics, in the broadest sense of this polysemous word, whereas no connection can be found between Jews and Pagans in the sources. The last book of the Visigothic law code cracks down at the same time on heretics and Jews, maybe due to the fact that, in the second half of the seventh century, both are equally viewed as flawed Christians. 
La question du sens des mots rencontrés dans les sources est au cœur de la pratique de l'historien moderne: non seulement le langage évolue à travers le temps, mais un même terme, dans un contexte précis, peut s'interpréter suivant différents sens, techniques ou d'usage courant, qu'un auteur peut même combiner intentionnellement de manière plus ou moins subtile. C'est certainement là que réside l'un des gisements majeurs de renouvellement de la recherche sur les sources du très haut Moyen Âge. Tenter de définir le «peuple» juif des premiers siècles médiévaux peut ainsi passer par un examen attentif du langage des textes qui l'envisagent comme tel. Nous aborderons ici la problématique identité juive, pour le monde visigothique, à travers deux médiations, celle, universelle, du discours, et celle de l'extériorité de nos sources, produites par la société majoritaire où sont immergés les juifs hispaniques. Après avoir examiné si la terminologie qu'elles emploient renvoie plutôt à une communauté religieuse ou à un groupe ethnique, nous aborderons les rapports qu'elles établissent ou suggèrent entre les juifs et d'autres dissidents religieux du temps, païens et hérétiques.

\section{LA DÉSIGNATION DU GROUPE JUIF DANS LES SOURCES CHRÉTIENNES VISIGOTHIQUES}

Une recherche systématique dans les bases de données numériques montre que les sources hispaniques des $\mathrm{V}^{\mathrm{e}}$-VII ${ }^{\mathrm{e}}$ siècles désignent le groupe des juifs par des termes aussi bien civiques ou ethniques (populus, plebs, gens, natio) que par des caractérisations religieuses (secta, superstitio).

\section{Un peuple?}

S'il conviendrait en théorie de distinguer les désignations ethniques, gens et natio, de celles qui ont un contenu politique, ou plus précisément civique, comme populus et plebs, en pratique, cette distinction sémantique est loin d'être toujours claire en latin 
tardif. Certes, conformément à la tradition scripturaire ${ }^{1}$, les auteurs latins chrétiens opposent habituellement les juifs aux nationes ou aux gentes, et parlent dans ce cas plus volontiers du populus iudaeorum: les juifs viennent alors occuper la place conceptuelle des Romains, seul populus face à la multiplicité des nations. Ce choix terminologique est toutefois loin d'être systématique, et il ne faut surtout pas le considérer comme une règle : un même auteur, dans une même œuvre, peut désigner les juifs par les mots plebs, populus, gens ou natio, en opposant ces deux derniers termes au populus christianus, voire aux populi gentium ${ }^{2}$. Deux facteurs peuvent se combiner ici: la tendance du latin tardif à éroder parfois les sens spécifiques de termes voisins mais non exactement synonymes, qui admettent désormais d'être employés comme synonymes purs ${ }^{3}$; l'influence de l'Aduersus Iudaeorum de Tertullien, dont les premières lignes défendent l'emploi indifférencié de gens et de populus pour désigner le peuple juif ${ }^{4}$, à partir de l'exégèse des passages de la Genèse ${ }^{5}$ où l'Éternel promet que du sein de Rébecca naîtront deux peuples à travers Esaï et Jacob. Tertullien commence par gloser duo populi et duae gentes essent processurae ( «que deux peuples, deux nations en sortiraient ») en utilisant le système d'oppositions habituel Iudaei / gentes ( les nations, c'est-à-dire nous », ajoute-t-il). Mais dès la phrase suivante il emploie gens dans un sens différent (et singulier) pour revendiquer son équivalence à populus dans la désignation des uns et des autres, «afin d'éviter qu'aucun d'eux ne revendique pour soi, à partir d'une dénomination, le privilège de la grâce ». Tertullien, non pas le fondateur du latin chrétien, mais du moins notre premier témoin littéraire de cette langue ${ }^{6}$, pose ainsi les bases conceptuelles qui permettront désormais aux auteurs de parler aussi bien de la gens des juifs que du populus des juifs.

1. Olegario García de la Fuente (1994), p. 45.

2. Sondage réalisé dans l'Expositio Psalmorum de Cassiodore (VII, 176 ; XIII, 24 ; XIII, 209 ; XIX, 29 ; XLVII, 63).

3. Charles H. Grandgent (1907), p. 31 ; Veikko Väänänen (2012), § 207-208. Le caractère flottant de la terminologie tardive n'est pas limité au cas des juifs, et les Romains peuvent aussi s'autodésigner comme gens (Ralph Mathisen (2006), p. 1021, n. 86).

4. Tertullien, Aduersus Iudaeos, I, 3-4.

5. Gn 22, 17-18 et 25, 23.

6. Olegario García de la Fuente (1994), p. 359. 
En raison de l'attention toute particulière qu'il prête au langage, de l'importance de son œuvre et de son poids comme autorité tout au long du Moyen Âge, le premier auteur hispanique à interroger est Isidore de Séville. Dans les premières lignes du livre VIII des Étymologies, consacré aux religions, il introduit les juifs comme un populus ${ }^{7}$. Il les désigne de la même manière dans le De Ortu et Obitu Patrum, une suite de vies de personnages de la Bible destinée à l'enseignement, et dans ses œuvres exégétiques en général. Ses Questions sur l'Ancien Testament font exception: dans la première partie In Genesim, les juifs sont comptés au nombre des gentes soumises par les Romains, bien qu'ils se distinguent de tous les autres populi et gentes par leur Loi et la circoncision, qu'ils ont conservées intactes sous le régime païen, puis chrétien ${ }^{8}$. Il semble clair que, dans sa pratique d'écriture, Isidore suit les traces de Tertullien et considère que les deux termes peuvent être synonymes et neutres, c'est-à-dire étrangers à un système d'opposition entre juifs et nations. On peut aller plus loin et assurer que, même lorsqu'il emploie l'expression populus Iudaeorum, il a en vue un sens ethnique plus caractéristique du mot gens que du sens classique de populus. Revenons aux Étymologies et à la définition qu'il y donne de la gens :

La gens est une multitude issue d'un même principe, ou bien qui s'est détachée d'une autre nation suivant un mode de rassemblement propre (propriam collectionem), comme en Grèce, en Asie. C'est pourquoi on l'appelle aussi gentilitas. On dit gens à cause des générations de familles, car le mot vient du verbe « engendrer », comme «nation » du verbe « naître ${ }^{9}$.

Selon Isidore, l'unité originelle de l'humanité, à travers la descendance des trois fils de Noé, s'est d'abord divisée en 72 langues lors de la dispersion consécutive à Babel, puis en beaucoup plus de gentes, dont certaines parlent donc la même langue mais n'en sont pas moins distinctes, et qui sont cimentées par un principe commun ${ }^{10}$ (la collectio propria). Dans la suite $\mathrm{du}$ chapitre, il énumère les patriarches bibliques et les peuples

7. Isidore de Séville, Etymologiae, VIII, 1, 7.

8. Isidore de Séville, Quaestiones in Vetus Testamentum: In Genesim, VI, 17 , col. 226

9. Isidore de Séville, Etymologiae, IX, 2, 1.

10. Sur les critères distinguant les différentes gentes, cf. Hans-Werner Goetz (2003), p. 44. 
auxquels chacun d'eux a donné naissance, au nombre desquels se trouvent les Hébreux, descendants d'Heber, également appelés «Israélites » d'après le nouveau nom reçu par Jacob, et dont certaines tribus reçurent, après la scission des deux royaumes, le nom de «Judéens » ou « Juifs $»^{11}$. Il semble clair, suivant cette explication, que les juifs sont bien l'un des peuples entre lesquels la terre habitée a été répartie par ramifications successives. Leur définition ne peut être qu'ethnique, dans la mesure où pour Isidore les religions (traitées au livre précédent des Étymologies) ne sont pas un élément de subdivision des hommes : elles n'ont pas leur place dans la grande généalogie qui lui sert à décrire l'humanité, tant diachroniquement que synchroniquement ${ }^{12}$. Les religions, par le biais des conversions, ont toutes vocation à se fondre dans l'unité catholique de l'Ecclesia en construction; pas les gentes, qui resteront distinctes dans la suite des siècles. Ce point est illustré par un autre passage des Étymologies qui présente dans les mêmes termes, à quelques lignes de distance, la conversion des Goths et celle des juifs, établissant ainsi un parallèle évidemment voulu $^{13}$. Le chapitre V, 39, De descriptione temporum, constitue une sorte de brève chronique universelle insérée dans l'encyclopédie. Ses deux derniers paragraphes indiquent respectivement que, l'année 21 de l'empereur Maurice, les Goths sont devenus catholiques (catholici efficiuntur), et que la cinquième année du règne d'Héraclius, les juifs en Hispanie sont devenus chrétiens (christiani efficiuntur). La contiguïté chronologique de deux conversions aussi importantes (pour aussi illusoire que nous paraisse la seconde) évoque d'abord, pour l'auteur et le lecteur du $\mathrm{VII}^{\mathrm{e}}$ siècle, l'approche de la fin des temps; mais, combinée au choix de termes identiques, elle met pour nous en évidence qu'Isidore place juifs et Goths sur le même plan. Les juifs sont une gens, au même titre que les peuples barbares qui ont pénétré dans l'Empire romain et y ont construit des regna. Néanmoins, tout comme les Romains, ils ne méritent plus d'être placés au centre

11. Isidore de Séville, Etymologiae, IX, 2, 51-53.

12. Sur les deux axes diachronique et synchronique autour desquels se distribuent les gentes chez Isidore, en particulier dans ses Chroniques, cf. Jamie Wood (2013), p. 125-168.

13. Sur la possible portée de ce parallèle pour Sisebut lui-même et ses contemporains, cf. Céline Martin, Capucine Nemo-Pekelman (2008), p. 236. 
de l'histoire : la gens qui occupe désormais cette position est celle des Goths ${ }^{14}$.

Un argument d'ordre théologique incite par ailleurs les auteurs chrétiens, et en particulier ceux qui m'occupent ici, à définir le groupe juif comme un groupe ethnique, uni par des liens de sang et descendant d'un même ancêtre, et non comme un groupe religieux. La clef s'en trouve chez Hildefonse de Tolède, qui entreprend de définir la double appartenance de Marie en opposant son origine (juive) à sa foi (chrétienne), c'est-à-dire en distinguant, chez elle, la chair de l'esprit, dans un style synonymique caractéristique de certains auteurs visigothiques qui nous permet de situer sans équivoque les mots gens et populus appliqués aux juifs :

Que dis-tu, Juif, que proposes-tu, que cherches-tu à prouver, à opposer, à objecter? Notre Vierge que voici est tienne par la souche, tienne par l'extraction, tienne par la racine, tienne par la lignée, tienne par la race (gens), tienne par le peuple (populus), tienne par la nation, tienne par l'origine. Et pourtant elle est nôtre par ce qu'elle confesse, nôtre par ce qu'elle croit, nôtre par ce qu'elle accepte, nôtre par ce qu'elle révère $[\ldots]^{15}$.

Tant au sein de l'histoire des hommes que du point de vue de l'interprétation des Écritures, les auteurs chrétiens situent toujours les juifs dans la corporéité, la matière, la chair, alors qu'eux-mêmes affirment relever de l'âme et du spirituel. Cette dualité est exprimée dans la personne de Marie, fille d'un peuple et mère d'une religion. L'identité collective juive ne peut donc être qu'ethnique, fruit des liens du sang, alors que c'est un ciment immatériel qui unit les chrétiens entre eux.

\section{Une religion?}

Il n'en est pas moins vrai qu'on trouve également à plusieurs reprises, dans les sources normatives visigothiques, des mentions de la secta iudaica ${ }^{16}$ ou de la superstitio des juifs. Ces termes dessineraient-ils un groupe juif entendu comme une communauté de religion? Ainsi, une loi de Sisebut de $612^{17}$ punit de mort tout

14. Jamie Wood (2013), p. 148 sq.; Eleonora Dell'Elicine (2013), p. 166-180.

15. Hildefonse de Tolède, De uirginitate Sanctae Mariae, p. 165.

16. Ce n'est pas une nouveauté, car les juifs apparaissent déjà comme secta dans le Code théodosien. Cf. Ralph Mathisen (2014).

17. $L V$ XII, 2, 14. 
juif qui circoncirait un chrétien ou ferait passer une chrétienne «dans sa secte ou son rite». Une autre loi d'Ervige de $681^{18}$ condamne à des coups et à l'exil tout juif qui oserait insulter ou attaquer la religion chrétienne ou «défendre l'inanité de sa secte». Le canon conciliaire de 633 contre les unions mixtes ${ }^{19}$ impose aux enfants qui en sont issus de suivre « la religion chrétienne et non la superstition judaïque ». Cinq ans plus tard, un autre $\operatorname{canon}^{20}$ affirme que le roi Chintila a décidé de mettre fin aux «transgressions et aux superstitions » des juifs. Le mot superstitio apparaît également dans une loi d'Ervige ${ }^{21}$ et dans le placitum (engagement écrit pris sous la contrainte ${ }^{22}$ ) attribué à Braulio de Saragosse engageant à l'orthodoxie les juifs baptisés de force ; enfin le nouveau placitum de même objet intégré par Ervige à sa version du Liber Iudiciorum ${ }^{23}$ fait usage des deux termes secta et superstitio.

Le mot secta est traduit par «secte» suivant une solution de facilité qui permet de rappeler le mot latin correspondant. En réalité il indique le «fait de suivre (sequi) », c'est-à-dire un mode de vie, un système de principes guidant la conduite. Il est associé, dans la première loi citée, au mot voisin ritus; on peut aussi le rapprocher de conuersatio (manière de vivre) qui apparaît dans $L V$ XII, 3, 7, opposé à superstitio. Souvent utilisé pour désigner les hérétiques (secta arriana), exceptionnellement aussi les catholiques eux-mêmes ${ }^{24}$, le mot secta n'en vient en fait à qualifier un groupe de personnes que par métonymie, tout comme ritus ici : « s'il fait passer une chrétienne dans sa secte et son rite ${ }^{25}$ ».

Comme secta, le mot superstitio fait référence au culte, au choix religieux des juifs. La définition qu'Isidore donne de superstitio dans les Étymologies mérite d'être mentionnée :

La superstition est ainsi appelée parce que c'est une observance superflue ou «superinstituée ». D'autres la rattachent aux vieillards

18. $L V$ XII, 3,9 .

19. Conc. Tol. IV, c. 63.

20. Conc. Tol. VI, c. 3.

21. $L V$ XII, 3,7 .

22. Isidore de Séville, Etymologiae, V, 24, 18-19 pour la définition du mot et son rapport avec pactum.

23. $L V$ XII, $3,14$.

24. Cf. la constitution d'Honorius de 408 (C.Th. XVI, 5, 42) : « eos, qui ex catholicae sectae sunt inimici, intra palatium militare prohibemus ».

25. «In suam sectam ritumue transduxerit » (LV XII, 2, 14). 
qui ont survécu (superstites) de nombreuses années et, à cause de l'âge, sont en proie au délire et à une sorte de superstition, ne sachant pas quelles choses anciennes vénérer ni lesquelles adopter, dans leur ignorance des choses du passé ${ }^{26}$.

Observance religieuse inopportune, propre à des vieillards séniles, fils d'un autre âge, la superstitio dépasse bien sûr de beaucoup la portée de sa traduction dans une langue moderne. Contrairement à ce que l'on pourrait penser, son application aux juifs n'implique aucune équivalence avec le paganisme, car les sources visigothiques qui nous sont parvenues ne l'utilisent jamais pour qualifier les païens ${ }^{27}$. Attesté dans les sources latines dès le $\mathrm{III}^{\mathrm{e}}$ siècle avant notre ère, le terme a logiquement connu de nombreuses variations de sens; il en combine simultanément plusieurs dans l'Antiquité tardive, ce qui le rend dès lors intrinsèquement ambigu ${ }^{28}$. Visant suivant les contextes des cibles variables, chrétiens, païens, juifs ou hérétiques, mais toujours de manière péjorative, il met en valeur le caractère inadéquat de la religion incriminée : à partir de l'époque théodosienne, sa fonction est précisément d'éviter de la qualifier de religio, mot qui est très rarement utilisé au pluriel. La religio est la manière correcte de rendre un culte à Dieu, par définition unique ${ }^{29}$, la superstitio est son contraire ${ }^{30}$.

En résumé, secta et superstitio sont des qualificatifs religieux appliqués aux juifs, mais ils ne se trouvent pas au fondement de la définition de leur groupe. Résultant d'un choix erroné, ils en sont un attribut, mais un attribut secondaire, qui n'intervient qu'a posteriori, le groupe juif existant déjà comme tel. La

26. Isidore de Séville, Etymologiae, VIII, 3, 6.

27. Céline Martin (2015), p. 278.

28. Pour une étude exhaustive, cf. Michele Salzman (1987).

29. Il est vrai que l'interpretatio, souvent citée, de la constitution d'Arcadius et Honorius reprise au Bréviaire (BA, II, 1, 10) mentionne « les anciens [maiores] de leur religio [i.e des juifs] », ce qui laisse supposer l'existence d'une pluralité de religiones; toutefois sa dernière phrase montre que, pour le rédacteur, religio est synonyme de lex (il y est question des « anciens de leur loi »). Or le fait qu'il y ait plusieurs lois est une évidence pour les Romains, et la polysémie des termes qu'il manie, un aspect que l'historien ne devrait jamais négliger. La constitution II, 1, 10 à laquelle se réfère cette interpretatio mentionne, quant à elle, la superstitio des juifs, non leur religio...

30. Cette opposition traditionnelle est rappelée par Isidore de Séville dans ses Différences : «religio autem est in uirtute, superstitio uero in cultu inlicito » (De differentiis, I, 16). Cf. Jacques Fontaine (1989). 
caractérisation religieuse des juifs, certes envisagée collectivement dans les sources chrétiennes, n'apparaît pas non plus irréversible, mécaniquement subordonnée à la définition du groupe lui-même ${ }^{31}$. Isidore considère les juifs comme destinés à la conversion (dont il croit avoir été témoin au cours de sa vie) par leur nom même ${ }^{32}$ : la confession juive peut varier au cours de l'histoire, elle n'est donc pas un élément d'identité essentiel pour eux. Un passage de ses Sentences illustre ce caractère subordonné et second. Isidore y affirme que le mot «fils » peut se référer à la naissance (natiuitas), mais aussi à l'imitation (imitatio) : ainsi les juifs sont fils d'Abraham selon la chair, mais fils du diable selon le mode de vie (conuersatio), alors que les véritables fils d'Abraham sont ceux qui imitent sa foi, non ceux qui sont issus de sa chair: les chrétiens. Les juifs ont en définitive fait le choix opposé à celui qu'Hildefonse de Tolède attribue à Marie ${ }^{33}$, et c'est ce choix religieux, qui les rapproche des autres non-catholiques, qui fonde leur condamnation.

\section{LES RAPPORTS ENTRE LES JUIFS ET LES AUTRES NON-CATHOLIQUES}

Quel type de relation les sources chrétiennes hispaniques établissent-elles entre les juifs et les autres non-catholiques? On a constaté précédemment l'existence, pour Isidore de Séville, d'un réel parallèle entre la conversion des Goths ariens en 589 et la supposée conversion des juifs hispaniques en 615. Revenons à présent sur les descriptions systématiques que cet auteur a proposées, non plus de la géographie et de l'histoire des peuples, mais de l'organisation religieuse de l'humanité. On trouve la principale dans ses Étymologies, au livre VIII, De Ecclesia et

31. Cf. Benjamin Isaac (2004) pour une distinction, en ce sens, entre racisme et préjugé ethnique.

32. L'étymologie qu'il propose de Judaei et de Hebraei (Etymologiae, VIII, 4,1-2) s'écarte de sa source, Jérôme, précisément pour mettre en valeur l'éminente convertibilité des juifs. Cf. Angelo Valastro Canale (2000), p. 59-60.

33. Ce « choix », qui implique la rupture de l'Alliance avec Dieu, a précisément lieu, pour Isidore, au moment de l'Incarnation (Jamie Wood (2013), p. 138), c'està-dire lorsque le personnage marial intervient, non pas à la destruction du Second Temple. 
sectis. Les cinq premiers chapitres en sont consacrés aux juifs et aux chrétiens, les six autres aux païens, en deux blocs clairement différenciés ${ }^{34}$. Les titres du premier bloc suggèrent trompeusement une équivalence entre chrétiens et juifs :

1. De ecclesia et synagoga

2. De religione et fide

3. De haeresi et schismate

4. De haeresibus iudaeorum

5. De haeresibus christianorum

En réalité le premier chapitre concerne essentiellement l’Église, ne mentionnant en passant la Synagogue que comme un terme que les juifs se sont en quelque sorte réservé. Le chapitre II concerne la religion chrétienne (rappelons qu'il n'existe qu'une religio et qu'une fides), le chapitre III concerne les divisions internes à l'Église, hérésies, schismes, sectes et superstitions ; le chapitre IV consacre deux phrases aux juifs en général, puis énumère leurs différentes sectes, qu'Isidore appelle " hérésies », conformément à la tradition grecque ${ }^{35}$; enfin le chapitre $\mathrm{V}$ s'occupe des hérétiques «qui se sont écartés de l'Église ${ }^{36}$ », ce qui n'est évidemment pas le cas de ceux du chapitre IV, qui n'en ont jamais fait partie. L'utilisation du même terme dans deux sens différents doit d'ores et déjà attirer notre attention sur sa polysémie, sur laquelle nous allons revenir. Voici la définition qu'il en donne :

L'hérésie vient de «choix » en grec, de ce que chacun choisit pour lui-même ce qui lui paraît le mieux, comme les philosophes péripatéticiens, académiciens, épicuriens et stoïciens, ou comme ceux qui, méditant un dogme pervers, sont sortis de l'Église de leur propre chef. C'est pourquoi l'hérésie est désignée par le mot grec traduit par « choix », car par elle chacun choisit soi-même, de sa propre décision, toute chose qu'il veut établir ou adopter pour soi.

Isidore a bien conscience qu'il existe deux sortes d'hérétiques, ceux qui adhèrent aux différentes écoles philosophiques, auxquelles il assimile «les sectes juives » (le sens le plus ancien), et les hérétiques « sortis de l'Église » (le nouveau sens, attesté depuis

34. Jacques Fontaine (1989), p. 395.

35. Voir le célèbre passage de Flavius Josèphe qui les énumère (La Guerre des Juifs, II, 8, 2).

36. «Quidam etiam heretici, qui de Ecclesia recesserunt » (Isidore de Séville, Etymologiae, VIII, 5, 1). 
Lactance). Leur point commun, également négatif à ses yeux, est de « choisir pour eux-mêmes » une doctrine, alors que le chrétien orthodoxe s'en remet pour cela aux Apôtres, qui eux-mêmes ne font que transmettre, sans choix personnel, la doctrine enseignée par le $\mathrm{Christ}^{37}$.

Une autre œuvre où Isidore dresse un tableau religieux de l'humanité est le premier livre des Sentences. Dans son chapitre XVI, De Ecclesia et heresibus, il oppose l'Église, répandue à travers le monde, aux hérésies, qui «se trouvent soit dans un coin particulier du monde, soit dans un seul peuple $(\text { gens })^{38} »$. Ce particularisme, qui ne concerne pas les païens, est un premier aspect qui rapproche l'hérésie chrétienne du judaïsme ${ }^{39}$. Un autre point commun est qu'un païen égaré peut commettre l'erreur de se convertir à l'une ou à l'autre, mais qu'Isidore n'envisage pas de conversions suivant d'autres combinaisons :

Ceux qui passent de l'idolâtrie au judaïsme ou à l'hérésie, comme le dit le prophète, «tombent d'un mal dans un autre et ne connaîtront pas le Seigneur », car ils sont passés de l'erreur de l'infidélité dans une autre erreur.

La symétrie entre les différentes confessions non-catholiques n'est donc pas totale, et si un rapprochement est possible, c'est celui qui associe judaïsme et hérésie.

A contrario, ni Isidore de Séville ni nos sources en général ne semblent associer la religion juive au paganisme. On a déjà vu que le mot superstitio appliqué aux juifs n'est pas significatif de ce point de vue, et que, dans son organisation, le livre VIII des Étymologies prend soin de séparer chrétiens et juifs d'un côté, païens de l'autre. Pourtant un historien a présenté il y a quelques années la thèse contraire $^{40}$ à propos d'une occurrence isolée. Wolfram Drews affirme en effet que le canon 10 du concile de Séville perdu ${ }^{41}$,

37. «Apostolos Dei habemus auctores, qui nec ipsi quicquam ex suo arbitrio, quod inducerent, elegerunt, sed acceptam a Christo disciplinam fideliter nationibus adsignaurunt » (Ibid., VIII, 3, 3).

38. Isidore de Séville, Sententiae, I, 16, 6.

39. Point signalé par Wolfram Drews (2002), p. 197.

40. Wolfram Drews (2002).

41. Il s'agit d'un canon conservé dans une collection systématique de vingt extraits conciliaires qui a été insérée dans la recension Juliana de l'Hispana. Plusieurs traitent des juifs, dont notre canon 10, qui est daté entre 615 (ou plus probablement 619) et 621 . 
qui reproche aux juifs baptisés de force de soustraire leurs propres enfants au baptême, les traite de païens, déployant ainsi un « extrémisme terminologique» inhabituellement agressif pour l'époque, car, comme il le signale à juste titre, s'il n'est pas rare que les juifs soient rapprochés des hérétiques, d'ordinaire la frontière entre eux et les païens est nettement tracée. Il s'agirait, à son avis, d'une stratégie rhétorique destinée à justifier le baptême forcé perpétré par Sisebut. Je crois pour ma part que le mot paganus ne doit pas être traduit ici par «païen », mais que, inséparable du verbe retinere, il a plutôt le sens de «profane, étranger à la religion » qui semble avoir existé en latin dès avant que les chrétiens ne reprennent le terme à leur compte ${ }^{42}$ :

Nous avons appris que certains juifs récemment appelés à la foi du Christ, par une ruse perfide, présentent au sacro-saint baptême d'autres enfants à la place des leurs, de sorte que, les faisant passer pour leurs enfants, ils leur font subir un double baptême, tandis que par une feinte secrète et criminelle, ils retiennent leurs rejetons hors de la religion (paganos retinent), car ils sont vraiment tous dès l'origine parjures par leur perfidie et jamais intégrés dans la foi ${ }^{43}$.

Il se trouve que le mot pagani est, généralement parlant, très peu fréquent dans les sources visigothiques ${ }^{44}$. On le rencontre essentiellement chez Isidore de Séville (auteur intellectuel probable du canon qui nous occupe), appliqué, certes, aux païens et non au domaine profane, comme c'est le cas selon nous ici. Ce dernier emploi de paganus n'a pas attiré l'attention des chercheurs et il est de toute évidence rare, une rareté que Constans attribuait au caractère "vulgaire » et provincial de l'acception, qui dissuadait les auteurs d'en faire usage. Quoi qu'il en soit, Isidore utilise avant tout paganus dans ses œuvres historiques, pour se référer à des personnages du passé, en particulier à propos de la fin de l'Empire

42. Léopold-Albert Constans (1918). À ma connaissance, les études ultérieures du mot paganus, qui présentent notamment les deux propositions alternatives de sens originel de «paysan » et « civil», n'ont pas repris l'idée de Constans, pourtant solidement étayée, quoique dans les limites d'un compte-rendu d'ouvrage. En l'occurrence c'est celle qui explique le mieux l'étrange hapax qui nous occupe.

43. Excerptum canonicum, 20: Ex concilio Spalitano capitulum decimum, Colección canónica hispana, éd. G. Martínez Díez, F. Rodríguez, V, Madrid, 1992.

44. Céline Martin (2015). 
romain ${ }^{45}$, ou pour se référer à une manière ancienne de penser ${ }^{46}$. Or selon lui les païens, dans le passé de l'histoire des hommes, n'ont jamais été que des chrétiens en puissance : «ils n'ont pas encore la foi $»^{47}$. Lorsque les autres sources visigothiques du VII ${ }^{\mathrm{e}}$ siècle continuent de mentionner le paganisme, elles font état de croyances ou de pratiques incorrectes qui proviennent ex gentibus ${ }^{48}$, parlent de l'idolatria ou des cultores idolorum ${ }^{49}$, mais n'utilisent plus le mot paganus. Le fait que les juifs ne soient jamais ailleurs dans les sources visigothiques traités de pagani ${ }^{50} \mathrm{~s}^{\text {'explique tout }}$ simplement parce qu'on n'y rencontre pratiquement jamais ce mot. Le recours à un emploi peu fréquent pour un mot devenu lui-même rare n'est finalement pas si choquant, surtout si l'on tient compte du fait qu'Isidore, son principal utilisateur au sein de notre corpus, fait justement profession de récupérer et ressusciter des mots sortis de l'usage.

Nous pouvons finalement retenir que, en dehors d'une association générique classique des trois ennemis du catholicisme, les sources visigothiques ne rapprochent jamais spécifiquement les juifs des païens, alors qu'elles les associent à l'occasion aux hérétiques. C'est tout particulièrement le cas dans la législation civile qui nous occupera pour terminer.

Le code de lois visigothiques, le Liber Iudiciorum, promulgué en 654 par le roi Recceswinth puis à nouveau par Ervige en 681, comprend douze livres et non seize comme le Code théodosien. Le dernier d'entre eux, comme le livre XVI du Théodosien, paraît réservé aux questions religieuses; néanmoins un examen attentif montre que la ressemblance n'est qu'apparente, car son premier chapitre, De temperando iudicio et remouenda pressura, bref et hétéroclite, traite du contrôle royal sur les juges et de la protection

45. Isidore de Séville, Historia Gothorum Vandalorum et Sueuorum, éd. MGH AA, XI, p. 272, 274 ; Id., Chronica, a. 344, a. 347 ; et bien sûr Id., Etymologiae, VIII, 10, 1.

46. Isidore de Séville, De differentiis, 219 ; Ibid., 498 ; Id., Etymologiae, $\mathrm{V}, 30,9$; etc.

47. « Nondum crediderunt» (Id., Etymologiae, VIII, 10, 2). Cf. Angelo Valastro Canale (2000), p. 80, et Jacques Fontaine (1989), p. 401 : « les païens sont eux-mêmes curieusement présentés comme des chrétiens qui s'ignorent ».

48. Profession de foi du roi Reccared lors du III ${ }^{\mathrm{e}}$ concile de Tolède (Colección canónica hispana, V, p. 72).

49. Conc. Tol. III, c. 16 ; Conc. Tol. XII, c. 11 ; Conc. Tol. XVI, c. 2.

50. Wolfram Drews (2002), p. 200. 
des justiciables. L'association avec les deux autres chapitres, qui ne rassemblent, eux, que des lois religieuses, doit certainement être comprise comme formant un tout servant à encadrer le rôle de souverain chrétien du roi visigothique ${ }^{51}$. Le deuxième chapitre a été compilé par les légistes de Recceswinth à partir d'un noyau de ses propres lois et de quelques autres plus anciennes. Le troisième est tout entier l'œuvre d'Ervige et a été ajouté en bloc lors de sa nouvelle promulgation du Liber; il suit de près l'organisation du chapitre II. Nous allons survoler l'ensemble des constitutions des chapitres II et III pour essayer d'en dégager la structure.

$L V$ XII, 2, 1 a une valeur de simple préambule programmatique dépourvu de portée juridique. Il s'agit d'une déclaration des devoirs du souverain en matière de conduite des hommes et de correction des mœurs, où Recceswinth se vante d'avoir jusque-là légiféré de manière à éviter les fautes des juifs et les excès des hérétiques. Il est vrai que, dans l'édition de Zeumer, le mot iudeorum (des juifs) est remplacé par iudiciorum (des juges), mais une récente étude de Liubov Chernina a montré que c'est la première variante, conservée dans les deux versions de l'édition de la Real Academia de 1815, qui est correcte $^{52}$. La phrase correspondante, qui ouvre la constitution, recouvre dès lors un sens cohérent: "Nous avons jusqu'ici dirigé des pas prudents sur les sentiers escarpés des fautes des juifs, et avancé avec une fermeté réglée à travers les excès glissants et immodérés de l'invention humaine (humane adinuentionis)... », où ces excès d'invention sont une allusion aux hérétiques, dont on sait qu'ils pèchent en décidant pour eux-mêmes, sans égard à l'auctoritas des Apôtres ${ }^{53}$. Cette loi introduit tout le chapitre XII, 2 et en précise la portée, qui s'étend donc tant aux hérétiques qu'aux juifs.

$L V$ XII, 2, 2 est consacrée aux hérétiques et uniquement à eux. Elle interdit de promouvoir des interprétations religieuses erronées par la controverse publique ou par l'écrit, et ne mentionne aucune

51. Sur la contextualisation eschatologique du mot pressura et de l'ensemble du livre XII, cf. Céline Martin (2015), p. 280.

52. Lex Visigothorum (2012), p. 771, et mon compte rendu de l'ouvrage dans la revue Initium (C. Martin, 2014).

53. Cf. supra n. 38. On trouve des parallèles à l'expression humana adinuentio visant les hérétiques chez Irénée de Lyon, Augustin (De haeresibus, 88, 7), Juste d'Urgel (Explicatio in Cantica Canticorum, 59), Martin de Braga (De superbia, VII)... 
pratique déviante (un point difficile à éluder si elle traitait des juifs), mais punit de manière différenciée les ecclésiastiques et les laïcs.

Le reste des lois du chapitre, de $L V$ XII, 2, 3 à XII, 2, 18, ne réprime que le judaïsme (la dernière est une novelle d'Égica insérée a posteriori). $L V$ XII, 2, 3 en explique la raison, avant de confirmer l'ensemble des lois anti-judaïques antérieures à Recceswinth: alors que la force divine (uirtus Dei) a fait disparaître toutes les hérésies, la seule souillure qui affecte le royaume est désormais le fait des juifs. Après avoir légiféré generaliter contre les hérétiques (allusion à la loi précédente, XII, 2, 2), Recceswinth confirme donc specialiter toutes les lois de ses prédécesseurs contre les juifs. Une telle formulation fait penser qu'il inclut ces derniers au nombre des hérétiques, ce qui n'est pas exceptionnel au $\mathrm{VII}^{\mathrm{e}}$ siècle, où le mot « hérétique » en est venu parfois à qualifier tous les non-orthodoxes ${ }^{54}$, sans perdre pour autant, en parallèle, son sens restreint de «chrétien sorti de l'Église». Toutes les lois suivantes ( $L V$ XII, 2, 4 à $8^{55}$ ) s'adressent aux juifs et conforment le groupe central du chapitre II. De construction identique, elles se composent d'un simple dispositif d'interdiction commençant par nullus ou nemo et renvoient à une sanction commune établie par $L V$ XII, 2, 11, la peine de mort de la main des autres juifs (ipsorum genti sue manibus). Suivent trois lois reprises des règnes antérieurs (Reccared et Sisebut) sur les esclaves des juifs, et une loi de Recceswinth réprimant la protection des juifs par les chrétiens ( $L V$ XII, 2, 15) qui se présente comme la conclusion de toutes les précédentes $^{56}$. La loi suivante, de son père Chindaswinth, punit aussi les chrétiens et non les juifs, cette fois en cas de pratiques judaïsantes.

Le chapitre III, entièrement dû à Ervige ${ }^{57}$, suit de près la structure du chapitre II, qu'il cherche à compléter et amplifier (il comprend 28 lois, contre 18 pour le précédent). Sa première loi passe en revue toutes les dispositions pour les confirmer

54. C'est le cas dans la législation justinienne : cf. Ralph Mathisen (2014).

55. Les lois 9 et 10 sont des lois de procédure de construction différente, probablement postérieures à 654 et insérées après coup dans le chapitre.

56. «In conclusionem omnium preteritarum legum » ( $L V$ XII, 2, 15).

57. Sur le libellus d'Ervige, cf. Céline Martin (2013), p. 231-232 et Ead. (2014), p. 62-64. 
ou les amender: ainsi XII, 2, 2 est amendée dans le sens d'une moindre sévérité pour les hérétiques qui se repentent ${ }^{58}$, et les lois suivantes du chapitre 2, « lois spéciales qui ont été établies contre les transgressions des juifs $»^{59}$, sont confirmées telles quelles, à l'exception d'une disposition sur les esclaves chrétiens et surtout de la peine de mort, qui est abolie (XII, 2, 11).

La deuxième loi d'Ervige, dirigée contre "les blasphémateurs de la Trinité », a pour cible les hérétiques et non les juifs, qu'elle ne nomme pas $^{60}$. Elle réprime le refus de la communion, le rejet des espèces sacrées et le blasphème contre la Trinité.

$L V$ XII, 3, 3 est un décret de baptême forcé, le second après celui de 615. Les lois suivantes du chapitre sont successivement des mesures d'interdiction diverses visant les juifs (4 à 9, 16 à 21), les chrétiens dans leurs rapports à eux $(10,22$ à 28$)$, leurs livres et leurs esclaves (11 à 13); il reprend également deux formules (placita) différentes d'engagement d'orthodoxie (14 et 15).

Les chapitres II et III du livre XII embrassent en définitive d'un même élan les dissidences hérétique et judaïque. Cela n'apparaît pas au premier coup d'œil, car si le titre de Recceswinth est explicite (De l'ablation de l'ensemble des erreurs de tous les hérétiques et des juifs), celui d'Ervige ne l'est pas: Sur les novelles des juifs, par où les anciennes sont confirmées et d'autres, nouvelles, sont ajoutées. Mais l'examen du contenu des lois ne laisse aucune place au doute: de manière exactement parallèle, la constitution qui ouvre chaque chapitre concerne à la fois juifs et hérétiques ; la suivante porte sur les hérétiques au sens étroit; toutes les autres traitent des juifs. La distinction opérée entre lois generales et speciales éclaire en outre la raison pour laquelle juifs et hérétiques peuvent être associés : le mot hérétique a plusieurs sens (on l'avait déjà constaté en consultant Isidore), et l'un d'eux englobe désormais les juifs. Peut-être s'agit-il moins de l'émergence d'un nouvel emploi du mot que d'un changement dans

58. L'amendement n'apparaît pas dans l'édition de Zeumer.

59. «Speciales leges, que in Iudeorum sunt transgressionibus posite» ( $L V$ XII, 3,1$)$. On a vu que Recceswinth distinguait sa deuxième loi anti-hérétique, generalis, des suivantes antijudaïques, speciales.

60. Contra, Bernhard Blumenkranz (1960), p. 301. En revanche P. D. King (1972), p. 130, n. 4 a bien vu qu'il s'agissait d'une norme anti-hérétique. La première législation connue contre le blasphème est l'œuvre de Justinien (nov. 77). 
la conception que se font les auteurs chrétiens de la confession juive, désormais envisagée comme une foi chrétienne défectueuse : les juifs hispaniques ont reçu le baptême, ils sont donc devenus chrétiens. Certes, les lois et les conciles tardifs reconnaissent à l'occasion que tous ne sont pas passés par les fonts baptismaux ; mais, tout comme les pagani du passé chez Isidore de Séville, les iudaei non baptisés du $\mathrm{VII}^{\mathrm{e}}$ siècle ne perdent rien pour attendre, ils sont des chrétiens en puissance. La langue prend acte de cet élan irrésistible de l'histoire des hommes en en faisant des hérétiques.

\section{Conclusion}

Pour nos sources, la judéité consiste donc en l'appartenance à un peuple, et ne saurait désigner autre chose. C'est pourquoi, baptisés et sincèrement convertis, les juifs n'en conservent pas moins leur appellation de iudaei, qui est une désignation ethnique et non religieuse, un paradoxe qui a été amplement commenté par les chercheurs. Le consensus selon lequel il n'y a pas d'antisémitisme au Moyen Âge, mais uniquement de l'antijudaïsme, a peut-être fait obstacle à son explication. Quoi qu'il en soit, il faut remarquer qu'Isidore a eu l'intuition d'un problème de ce point de vue, lorsqu'il affirme que tout comme les païens baptisés, «après avoir reçu la foi le juif ne peut plus être dit tel, comme l'atteste l'Apôtre Paul $»^{61}$. Ce remords terminologique n'a absolument pas été pris en compte par le reste de nos sources, ni d'ailleurs par luimême. Les juifs ne sont pas considérés comme titulaires d'une religion propre, mais de croyances et de pratiques religieuses défectueuses, dans un univers intellectuel où il n'existe qu'une seule religion. Pour corriger cette défectuosité, la démarche des catholiques hispaniques du VII ${ }^{\mathrm{e}}$ siècle est la même que celle qu'ils ont appliquée aux hérétiques, car les juifs ne sont pour eux, dans le fond, que des hérétiques au sens large.

$\mathrm{Y}$ a-t-il eu, en la matière, rupture historique lors du premier baptême forcé de 615 ? C'est très probable, mais il ne faudrait pas en déduire que les juifs sont alors passés d'un statut de religio licita à celui d'une simple hérésie du christianisme. L'expression religio

61. Isidore de Séville, Etymologiae, VIII, 10, 4. 
licita, contestée par les romanistes pour l'époque impériale ${ }^{62}$, ne bénéficie d'aucun appui textuel dans les sources visigothiques. La seule base que Wolfram Drews invoque en sa faveur ${ }^{63}$ est l'interpretatio du Bréviaire d'Alaric déjà mentionnée, qui concerne la procédure judiciaire (plus précisément, la possibilité d'avoir recours aux tribunaux juifs) et non l'exercice d'une religion ${ }^{64}$, et qui n'a donc aucune conséquence en matière de «légalité » du judaïsme.

Une autre rupture devrait plutôt attirer notre attention : celle de 694 , moment où les juifs hispaniques sont collectivement expulsés de la citoyenneté et réduits en esclavage ${ }^{65}$. C'est aussi le moment où les autorités visigothiques, tout en considérant que les juifs sont d'éternels " hérétiques », donc de mauvais chrétiens, impossibles à réformer (ce qui contraste avec l'optimisme d'un Isidore quelques dizaines d'années plus tôt ${ }^{66}$ ), en viennent à envisager les chrétiens de souche, qu'ils disent avoir été trahis par les juifs, comme un genus $^{67}$ ! En cette orée de VIII siècle, il semble que les catégories commencent à se brouiller et que la religion devienne transmissible par le sang. Peut-être toutes les confusions médiévales et modernes deviennent-elles alors possibles.

celine.martin@u-bordeaux-montaigne.fr

62. Capucine Nemo-Pekelman (2010), p. 79-114.

63. Wolfram Drews (2002), p. 193.

64. BA II, 1, 10, cf. supra n. 30.

65. Céline Martin, Capucine Nemo-Pekelman (2008) ; Céline Martin (2013) et Ead. (2014).

66. Cf. supra, n. 33.

67. Conc. Tol. XVII, tomus regius, éd. J. Vives (1963), p. 524. 


\section{SOURCES}

Braulio de Saragosse, Confessio uel professio Iudaeorum ciuitatis Toletanae, éd. Fidel Fita, Suplementos al Concilio Nacional Toledano VI, Madrid, 1881, p. 43-60.

CAssiodore, Expositio Psalmorum, éd. M. Adriaen, Magni Aurelii Cassiodori expositio psalmorum, Turnhout, Brepols (CCSL 97), 1958.

Colección canónica hispana, éd. Gonzalo Martínez Díez, Félix Rodríguez, vol. v, Madrid, 1992.

Concilios visigóticos e hispano-romanos, éd. J. Vives, Barcelone - Madrid, 1963.

Hildefonse de Tolède, De uirginitate Sanctae Mariae, éd. Valeriano Yarza Urquiola, Carmen Codoñer, Ildefonsi Toletani episcopi opera omnia, Turnhout, Brepols (CCSL 114A), 2007.

Isidore de SÉville, Chronica, éd. José Carlos Martín, Turnhout, Brepols (CCSL 112), 2003.

Isidore de Séville, De differentiis, éd. Carmen Codoñer, De differentiis (Diferencias) - Libro I. Introducción, edición crítica, traducción y notas, Paris, 1992.

IsIDORE DE SÉviLle, De ortu et obitu patrum, éd. César Chaparro Gómez, Isidorus Hispalensis. De ortu et obitu Patrum. Vida y muerte de los santos. Introducción, edición crítica y traducción, Paris, 1985.

IsIDORE DE SÉville, Etymologiae, éd. W. M. Lindsay, Oxford, 1911, dans Etimologías, trad. José Oroz Reta et Manuel-A. Marcos Casquero, Madrid (BAC 433-434), 1982-1983.

IsIDORE DE SÉville, Historia Gothorum Vandalorum et Sueborum, éd. MGH AA XI, p. 268-303, Berlin, 1894.

IsIDORE DE SÉville, Quaestiones in Vetus Testamentum seu Mysticorum expositiones sacrmentorum, éd. PL 83, col. 207-424.

Isidore de SÉville, Sententiae, éd. Pierre Cazier, Turnhout, Brepols (CCSL 111), 1998.

Leges Visigothorum, éd. Karl Zeumer, MGH Leges I, 1, Hanovre - Leipzig, 1902, p. 33-456.

Tertullien, Aduersus Iudaeos, éd. Hermann Tränkle, Q[uintus] S[eptimus] F[lorens] Tertullianus. Adversus Iudaeos. Mit einer Einleitung und kritischem Kommentar, Wiesbaden, 1964. 


\section{BibLiOgRAPHIE}

BLUMENKRANZ (Bernhard) : Juifs et chrétiens dans le monde occidental, 430-1096, Paris - Louvain, 2007 [Paris - La Haye, 1960].

Constans (Léopold-Albert): «Compte-rendu de JACQUes Zeiller. Paganus. Étude de terminologie historique », Journal des Savants 16-4 (1918), p. 213-214.

Dell'elicine (Eleonora): En el principio fue el Verbo. Políticas del signo y estrategias del poder eclesiástico en el reino visigodo de Toledo (589-711), Cadix, 2013.

Drews (Wolfram) : « Jews as Pagans? Polemical definitions of identity in Visigothic Spain », Early Medieval Europe 11-3 (2002), p. 189-207.

FontAine (Jacques): «Le 'sacré' antique vu par un homme du VII ${ }^{\mathrm{e}}$ siècle : le livre des Étymologies d'Isidore de Séville », Bulletin de l'Association Guillaume Budé: Lettres d'Humanité 48 (1989), p. 394-405.

GARcía DE LA Fuente (Olegario): Latín bíblico y latín cristiano, Madrid, 1994.

Goetz (Hans Werner): «Gens. Terminology and perception of the 'germanic' peoples from Late Antiquity to the Early Middle Ages », The construction of communities in the Early Middle Ages, éd. R. Corradini, M. Diesenberger, H. Reimitz, Leyde, 2003, p. 39-64.

GRANDGENT (Charles H.) : An introduction to vulgar latin, Boston, 1907. IsAAC (Benjamin): The Invention of Racism in Classical Antiquity, Princeton, 2004.

KING (P. D.) : Law and Society in the Wisigothic Kingdom, Cambridge, 1972.

Lex Visigothorum (Liber Iudiciorum). Textum latinum. Interpretatio rossica. Studia. Вестготская Правда (Книга Приговоров). Латинский текст. Перевод. Исследование, dir. O. V. Aurov, A. V. Marey, Moscou, 2012.

Martin (Céline) : "La degradación cívica de los judíos libres en el reino visigodo de Toledo », Marginados sociales y religiosos en la Hispania tardorromana y visigoda, éd. R. González Salinero, Madrid - Salamanque, 2013, p. 221-241.

Martin (Céline) : «Statut des juifs, statut de libre dans l'Occident du haut Moyen Âge: l'exemple ibérique », Jews in Early Christian Law. Byzantium and the Latin West, 6th-11th centuries, éd. J. V. Tolan, N. de Lange, L. Foschia, C. Nemo-Pekelman, Turnhout, 2014, p. 55-71. 
MARTIN (Céline) : « Compte rendu de Lex Visigothorum (Liber Iudiciorum). Textum latinum. Interpretatio rossica. Studia. Вестготская Правда (Книга Приговоров). Латинский текст. Перевод. Исследование, dir. O. V. Aurov, A. V. Marey, Moscou, $2012 »$, Initium. Revista Catalana d'Història del Dret 19 (2014), p. 251-258.

MARTIN (Céline) : «De sacrilegiis extirpandis. Interpretar la legislación contra el paganismo en la Hispania de los siglos VI-VII », La Iglesia como sistema de dominación en la Antigüedad Tardía, coord. J. Fernández Ubiña, Alberto J. Quiroga, P. Ubric Rabaneda, Grenade, 2015, p. 273-292.

Martin (Céline), Nemo-Pekelman (Capucine) : «Les juifs et la cité. Pour une clarification du statut personnel des juifs de l'Antiquité tardive à la fin du royaume de Tolède ( $\mathrm{IV}^{\mathrm{e}}-\mathrm{VII}^{\mathrm{e}}$ siècles) », Antiquité tardive 16 (2008), p. 223-246.

Mathisen (Ralph) : «Peregrini, Barbari, and Cives Romani Concepts of Citizenship and the Legal Identity of Barbarians in the Later Roman Empire », The American Historical Review, 111-4 (Oct 2006).

MAthisen (Ralph) : « The Citizenship and Legal Status of Jews in Roman Law during Late Antiquity (ca. 300-540 CE) », Jews in Early Christian Law. Byzantium and the Latin West, 6th-11th centuries, éd. J. V. Tolan, N. de Lange, L. Foschia, C. Nemo-Pekelman, Turnhout, 2014, p. 35-53.

Nemo-Pekelman (Capucine) : Rome et ses citoyens juifs. IV $V^{e}-V^{e}$ siècles, Paris, 2010.

Salzman (Michele R.) : «Superstitio in the Codex Theodosianus and the Persecution of Pagans », Vigiliae Christianae 41 (1987), p. 172-188.

V̈̈̈̈NÄNEN (Veikko) : Introduction au latin vulgaire, Paris, 2012 [1963]. Valastro Canale (Angelo): Herejías y sectas en la Iglesia antigua. El octavo libro de las Etimologías de Isidoro de Sevilla y sus fuentes, Madrid, 2000.

Wood (Jamie) : «Religiones and gentes in Isidore of Seville's Chronica maiora. The Visigoths as a chosen people », Post-Roman transitions : Christian and Barbarian identities in the early medieval west. Cultural Encounters in Late Antiquity and the Middle Ages (14), Turnhout, 2013, p. 125-168. 